\title{
Glutathione S-transferase, incense burning and asthma in children
}

\author{
I-J. Wang*,\#,ף, C-H. Tsai' ${ }^{+}$, C-H. Chen ${ }^{\S}$, K-Y. Tung ${ }^{+}$and Y.L. Lee Lef $^{+, f}$
}

ABSTRACT: Incense burning is a popular practice in many family homes and temples. However, little is known about the effects of indoor incense burning and genetic polymorphisms on asthma. This study evaluated the effects of indoor incense burning and glutathione S-transferase (GST) genetic polymorphisms on asthma and wheeze.

In 2007, 3,764 seventh-grade schoolchildren (mean \pm SD age $12.42 \pm 0.65$ yrs) were evaluated using a standard questionnaire for information about respiratory symptoms and environmental exposures. Multiple logistic regressions were performed to assess the association between GST polymorphisms and incense burning frequency on asthma and wheeze, after adjusting for potential confounders.

The frequency of incense burning at home was associated with increased risk of current asthma $(p=0.05)$, medication use $(p=0.03)$ and exercise wheeze $(p=0.001)$. GST01 (GSTT1) null genotypes were associated with current asthma (OR 1.43, 95\% $\mathrm{Cl} 1.00-2.04$ ) and medication use (OR 1.46, 95\% Cl 1.01-2.22). GSTT1 showed a significant interactive effect with incense burning on current asthma, current wheeze and nocturnal wheeze. The frequency of incense burning was associated with increased risk of current asthma, medication use, lifetime wheeze, nocturnal wheeze and exercise wheeze in an exposure-response manner among children with GSTT1 null genotype $(p<0.05)$.

Incense burning is a risk factor for asthma and wheezing, especially in GSTT1 genetically susceptible children.

KEYWORDS: Asthma, gene-environment interaction, GSTT1, incense burning

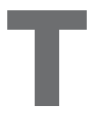

he prevalence of asthma in Asia differs from that in western countries [1, 2]. Culture, lifestyle and residential environment are all potential risk factors that contribute to such differences [3]. Incense burning and its effect on respiratory health is worth exploring because exposure to incense smoke may be comparable to exposure to tobacco smoke, as incense burning is a traditional and popular practice among many families and in temples [4,5]. In addition to burning huge amounts of incense in temples during major Chinese or religious festivals, many believers also burn incense when they worship at home or in temples.

The physical characteristics of the incenses are similar. Typical Chinese incense is composed of $35 \%$ fragrance material, 33\% bamboo stick, $21 \%$ herbal and wood powder by weight, and $11 \%$ adhesive powder [6]. The emitted smoke contains particulate matter (PM), diethylphthalate (DEP), gas products and many other organic compounds.
Due to its slow and incomplete combustion, incense burning produces continuous smoke that generates toxic gases and chemical particles, such as polycyclic aromatic hydrocarbons (PAHs), carbon monoxide, isoprene and benzene, that can easily accumulate inside houses with inadequate ventilation [6]. It is reported that PAHs and airborne PM from incense burning constitute potential health hazards, which are related to respiratory symptoms, asthma, elevated cord blood immunoglobulin (Ig)E levels, contact dermatitis and cancer $[7,8]$. Therefore, exploring the health effects of exposure to incense smoke in children is warranted.

The glutathione S-transferase (GST) superfamily includes a number of susceptibility genes. Several members, including GST $\mu 1$ (GSTM1), GST 01 (GSTT1) and GST $\pi 1$ (GSTP1), are expressed in the respiratory tract and involved in asthma pathogenesis, including oxidant defences, xenobiotic metabolism and detoxification of hydroperoxides [9].

\section{AFFILIATIONS}

*Dept of Paediatrics, Taipei Hospital Dept of Health

'School of Medicine, Fu Jen Catholic University,

+Institute of Epidemiology and Preventive Medicine,

${ }^{f}$ Research Center for Genes, Environment and Human Health, College of Public Health, National Taiwan University, Taipei,

\#College of Public Health, China Medical University, Taichung and §Guangfu Township Health Office, Hualien County Health Bureau, Hualien, Taiwan.

\section{CORRESPONDENCE}

Y.L. Lee

Institute of Epidemiology and

Preventive Medicine, College of

Public Health

National Taiwan University

Taipei 100

Taiwan

E-mail: leolee@ntu.edu.tw

Received:

Aug 262010

Accepted after revision:

Nov 012010

First published online:

Nov 252010 
The GSTT1 null genotype has been shown to be significantly associated with atopy [10]. Among GSTT1-deficient children, in utero exposure to smoke is also reportedly associated with significant decrements in lung function [11]. Furthermore, SAADAT and ANSARI-LARI [12] reported that both GSTT1 and GSTM1 null genotypes lack protection against asthma development in adults with a positive history of smoking. Evidence suggests that variants in the GSTM1 and GSTP1 loci may contribute to the occurrence of childhood asthma and increase susceptibility to adverse effects of air pollution [13]. Asthmatic children with GSTM1 null and GSTP1 $1^{\mathrm{Val} / \mathrm{Val}}$ genotypes also appear more susceptible to developing respiratory symptoms related to ozone exposure [14]. However, whether or not polymorphism of the GST genotypes can modify the effects of incense smoke on asthma requires investigation. Thus, the purpose of this study was to evaluate the association between indoor incense burning and GST polymorphisms in children.

\section{METHODS}

\section{Study population}

Between September and October 2007, 5,082 middle-school children were recruited from public schools in 14 Taiwan Children Health Study communities. 3,764 seventh-grade schoolaged children completed the standard questionnaire. Each student took home an informed consent form and a standard questionnaire to be answered by parents with additional questions concerning the effect of incense burning on asthma symptoms [15]. Questions in the questionnaire included basic demography, residential environmental factors (e.g. environmental tobacco smoke (ETS), pets and cockroaches at home, dampness of the house, fungus on the house walls, air cleaners, air conditioners, dehumidifiers, and carpets at home) and family history of atopic diseases. All of the selected subjects were of the same ethnic origin. The institutional review board (National Taiwan University Hospital Research Ethics Committee, Taipei, Taiwan; study number 200902042R) approved the study protocol, which is as previously described [15].

\section{Health effects assessment}

The parents' questionnaire responses were used to categorise children's asthmatic and wheezing status as previously described [15]. Children were considered as having lifetime asthma if the answer to the question "Has a doctor ever diagnosed this child as having asthma?" was "yes". Current asthma was defined as physician-diagnosed asthma with any asthma-related symptoms or illnesses in the previous 12 months. Medication use was defined as use of any inhaled, oral or intravenous medication in the previous 12 months. Lifetime wheeze was determined by a positive response to the question "Has your child ever had wheeze or whistling in the chest at any time in the past when he/she did not have a cold or the flu?" Those who reported attacks in the previous 12 months were identified as having current wheeze. Exercise wheeze was determined by a positive response to the question, "Has your child ever had wheeze or shortness of breath triggered by exercise?"

\section{Incense exposure}

Since children were exposed to incense smoke mostly in their homes, incense smoke dosage was determined from the question "How often is incense burned in the household when the child is in the room during the past 12 months?" The frequency of incense burning was divided into three categories: never, less than daily (two times per month or during major festivals) and daily (all day long, every day, and morning and night every day).

\section{Analysis of genetic polymorphisms}

Cotton swabs containing oral mucosa were collected and stored at $-80^{\circ} \mathrm{C}$ before analysis. Genomic DNA was isolated using the phenol/chloroform extraction method [16]. Genetic polymorphisms of the GSTM1 and GSTT1 genes were identified by PCR, while GSTP1 polymorphisms were detected by the PCRrestriction fragment length polymorphism method, as previously described [16]. The laboratory staff was blinded to each subject's clinical status. Genotype assignments were based on two consistent experimental results. About $15 \%$ of the randomly selected samples were sequenced and all were consistent with the initial genotyping results.

\section{Statistical analysis}

Multiple logistic regression models adjusted for major covariates were made to examine the effects of incense burning with GST genotypes on asthma and wheezing. To further assess gene-environment interaction, the combined association of incense burning and GST genotypes was examined by stratifying into four groups: no incense exposure with GSTT1 or GSTM1 present, no incense exposure with GSTT1 or GSTM1 null, incense exposure with GSTT1 or GSTM1 present, and incense exposure with GSTT1 or GSTM1 null. With regard to GSTP1, the genotypes were also stratified into four groups: no incense exposure with GSTP1 $1^{\text {Ile/lle }}$, no incense exposure with GSTP1 $1^{\text {Ile/Val }}$ or GSTP1 $1^{\text {Val/Val }}$, incense exposure with GSTP $1^{\text {Ile/lle }}$, and incense exposure with GSTP1 $1^{\text {Ile/Val }}$ or GSTP1 $1^{\text {Val/Val }}$. Bonferroni correction was used to address the problem of multiple comparisons.

Gene-environment interaction was tested by adding a product term in the regression model. For categorical variables with more than two categories, the gene-environment interaction was evaluated using the likelihood ratio test, comparing the model with indicator variables for the cross-classified variables with a reduced model containing indicator variables for the main effects only. Within genotype categories, a one degree of freedom trend test was used to evaluate the possible exposure-response relationship across categories of the incense burning variables.

Odds ratios and 95\% confidence intervals were adjusted for important potential confounders in all analyses. Selection of confounders that were included in the model was based on $a$ priori consideration and standard statistical procedure of a $10 \%$ change in point estimates. Subjects with missing covariate information were included in the model using missing indicators. All hypothesis testing was two-sided at the significance level of 0.05 and performed using the SAS software version 9.1 (SAS Institute Inc., Cary, NC, USA).

\section{RESULTS}

Of the 3,764 children with complete questionnaire and genotyping data enrolled in this study, 26 were excluded due to active smoking. Of the 3,738 participants included in the final analysis, $63.0 \%$ had residential incense smoke exposure. Table 1 provides the demographic characteristics of the study 


\begin{tabular}{|c|c|c|}
\hline \multirow[t]{2}{*}{ TABLE 1} & \multirow[b]{2}{*}{$\begin{array}{c}\text { With } \\
\text { genotyping }\end{array}$} & \multirow[b]{2}{*}{$\begin{array}{l}\text { All eligible } \\
\text { participants }\end{array}$} \\
\hline & & \\
\hline Subjects n & 3764 & 5082 \\
\hline \multicolumn{3}{|l|}{ Demographic information } \\
\hline Males & $1848(49.1)$ & 2464 (48.5) \\
\hline Age yrs & $12.26 \pm 0.50$ & $12.42 \pm 0.65$ \\
\hline \multicolumn{3}{|l|}{ Parental education ${ }^{\#}$ yrs } \\
\hline$\leqslant 12$ & $2319(62)$ & 3201 (63.4) \\
\hline $13-15$ & 740 (19.8) & $964(19.1)$ \\
\hline$\geqslant 16$ & $679(18.2)$ & $884(17.5)$ \\
\hline \multicolumn{3}{|l|}{ Gestational age $^{\#}$} \\
\hline$\leqslant 37$ & $335(9.1)$ & $461(9.3)$ \\
\hline$>37$ & $3342(90.9)$ & $4496(90.7)$ \\
\hline Parental asthma ${ }^{\#}$ & $114(3.1)$ & $140(2.9)$ \\
\hline Parental atopy ${ }^{\#}$ & $957(26.4)$ & $1263(25.8)$ \\
\hline \multicolumn{3}{|l|}{ Family income ${ }^{\#}$ TWD } \\
\hline$\leqslant 400000$ & $1249(35.8)$ & $1777(37.7)$ \\
\hline $400001-800000$ & $1408(40.4)$ & 1853 (39.3) \\
\hline$\geqslant 810000$ & 828 (23.8) & 1080 (22.9) \\
\hline Active smoking ${ }^{\#}$ & $26(0.7)$ & $37(0.7)$ \\
\hline $\begin{array}{l}\text { Maternal smoking during } \\
\text { pregnancy }{ }^{\#}\end{array}$ & $146(3.9)$ & $198(3.9)$ \\
\hline ETS at home ${ }^{\#}$ & $1774(47.4)$ & $2471(48.9)$ \\
\hline \multicolumn{3}{|l|}{ Incense burning at home ${ }^{\#}$} \\
\hline Never & $1326(37.0)$ & $1724(35.8)$ \\
\hline Less than daily & $1021(28.5)$ & $1398(29.0)$ \\
\hline Daily & $1237(34.5)$ & 1699 (35.2) \\
\hline \multicolumn{3}{|l|}{ Respiratory outcomes } \\
\hline \multicolumn{3}{|l|}{ Asthma } \\
\hline Lifetime asthma & $292(7.8)$ & $375(7.4)$ \\
\hline Current asthma & $114(3.1)$ & $140(2.9)$ \\
\hline Medication use & $96(2.6)$ & $123(2.4)$ \\
\hline \multicolumn{3}{|l|}{ Wheeze } \\
\hline Lifetime wheeze & $449(12.0)$ & $586(11.6)$ \\
\hline Current wheeze & $150(4.0)$ & $186(3.7)$ \\
\hline Nocturnal wheeze & 82 (2.2) & 109 (2.2) \\
\hline Exercise wheeze & $206(5.5)$ & $276(5.5)$ \\
\hline
\end{tabular}

Data are presented as $n(\%)$ or mean \pm SD, unless otherwise stated. TWD: New Taiwan dollar; ETS: environmental tobacco smoke. ${ }^{*}$ : number of subjects does not sum to total because of missing data.

population. The characteristics were almost identical between those with and without genotyping. The overall allele frequencies were $48.1 \%$ null GSTT1, 56.9\% null GSTM1 and 65.4\% GSTP1 Val105Ile polymorphisms.

The results showed that incense burning at home was associated with increased risk of current asthma (OR 1.36, 95\% CI 1.01-2.00; $\mathrm{p}=0.05)$, medication use (OR 1.52, 95\% CI 1.02-2.42; $\mathrm{p}=0.03$ ) and exercise wheeze (OR 1.64, 95\% CI 1.18-2.28; $\mathrm{p}=0.001$ ) (table 2). The GSTT1 null genotypes were associated with current asthma (OR 1.43, 95\% CI 1.00-2.04) and medication use (OR 1.46, 95\% CI 1.01-2.22). The GSTT1 null genotype had a positive relationship with wheeze that did not reach statistical significance (table 2). Both the GSTM1 null and GSTP1 $1^{\text {Ile/Ile }}$ genotypes were not significantly associated with asthma or wheeze.

As only the GSTT1 null genotype was associated with asthma, the association between incense burning and GSTT1 genotype on asthma and wheeze was further examined. In a mutually adjusted model of potential confounders, the GSTT1 null genotype showed a significant interaction with incense burning on current asthma, current wheeze and nocturnal wheeze $(\mathrm{p}<0.05)$ (table 3). However, analyses of GSTM1 and GSTP1 did not show significant interactive effects (online supplementary tables). Incense burning and GSTT1 had a synergistic effect on current asthma, with OR 1.40 (95\% CI 0.76-2.57) for no incense exposure with GSTT1 null genotype, OR 1.36 (95\% CI 0.77-2.40) for incense exposure with GSTT1 present genotype and OR 1.92 (95\% CI 1.11-3.32) for incense exposure with GSTT1 null genotype (table 3).

After stratification by GSTT1 genotype, the frequency of incense burning was associated with increased risk of current asthma $(p=0.04)$, medication use $(p=0.02)$, lifetime wheeze $(p=0.04)$, nocturnal wheeze $(p=0.003)$ and exercise wheeze $(p=0.01)$ in an exposure-response manner for children with the GSTT1 null genotype (table 4). However, there were no significant relationships between incense burning and GSTM1 and GSTP1 genotypes on asthma and wheeze (online supplementary tables).

\section{DISCUSSION}

This study is a contribution to the literature on the potential associations between genetic polymorphisms, incense burning and paediatric asthma. The combination effects of GSTT1 polymorphisms and incense smoke exposure on the risk of childhood asthma have not been previously studied. Asthma and wheezing are positively associated with the frequency of incense burning. In addition, children carrying the GSTT1 null genotype are most susceptible to the adverse effects of incense smoke.

The factors examined (age, sex, parental education, family income, parental history of atopy, gestational age, residence, maternal smoking, pre- and post-natal ETS exposure, pets and cockroaches at home, dampness of the house, fungus on the house walls, and carpets at home) may all confound the results. As such, they are all considered as potential confounders in this survey and those who had a $10 \%$ change in point estimates in the statistical procedures have been adjusted. Those with smoking habits have also been eliminated as study subjects to avoid the confounding effect of active smoking. In addition, the subjects were all Han Chinese, with the premise that they are rather homogeneous. Therefore, the confounding effect of ethnicity was lessened.

As the frequencies of incense burning are associated with increased risk of current asthma and medication use, instead of lifetime asthma, it is suspected that incense burning may play a more important role in exacerbating asthmatic symptoms among GSTT1 null children. Consistent with a previous epidemiological study, AL-RAWAS et al. [17] also report that incense burning is a common trigger of worsening of wheezing among asthmatic children, but is not associated with the prevalence of asthma. Furthermore, YANG et al. [18] have also discovered that incense burning and mosquito repellant 
TABLE 2 Effects of incense burning at home and glutathione S-transferase genotype on subcategories of asthma and wheeze

\begin{tabular}{|c|c|c|c|c|c|c|c|}
\hline & Lifetime asthma & Current asthma & Medication use & Lifetime wheeze & Current wheeze & $\begin{array}{l}\text { Nocturnal } \\
\text { wheeze }\end{array}$ & Exercise wheeze \\
\hline Incense burning & $1.05(0.80-1.36)$ & $1.36(1.01-2.00)$ & $1.52(1.02-2.42)$ & $1.10(0.88-1.37)$ & $1.07(0.75-1.53)$ & $1.31(0.8-2.14)$ & $1.64(1.18-2.28)$ \\
\hline \multicolumn{8}{|c|}{$\begin{array}{l}\text { Frequency of incense } \\
\text { burning }^{\#}\end{array}$} \\
\hline Less than daily & $0.76(0.54-1.07)$ & $1.13(0.70-1.83)$ & $1.29(0.72-2.30)$ & $0.92(0.70-1.21)$ & $1.00(0.64-1.56)$ & $1.07(0.58-2.01)$ & $1.62(1.09-2.41)$ \\
\hline Daily & $1.22(0.90-1.64)$ & $1.52(0.99-2.34)$ & $1.75(1.03-2.96)$ & $1.21(0.94-1.55)$ & $1.12(0.74-1.70)$ & $1.68(0.97-2.93)$ & $1.88(1.29-2.74)$ \\
\hline $\mathrm{p}$-value for trend & 0.20 & 0.05 & 0.03 & 0.13 & 0.60 & 0.06 & 0.001 \\
\hline GSTT1 null & $1.21(0.95-1.56)$ & $1.43(1.00-2.04)$ & $1.46(1.01-2.22)$ & $1.17(0.95-1.44)$ & $1.14(0.81-1.60)$ & $1.14(0.72-1.79)$ & $1.09(0.81-1.47)$ \\
\hline GSTM1 null & $0.87(0.68-1.12)$ & $0.89(0.63-1.27)$ & $0.84(0.55-1.27)$ & $0.98(0.80-1.21)$ & $0.91(0.65-1.27)$ & $0.73(0.47-1.16)$ & $0.90(0.67-1.21)$ \\
\hline GSTP1 ${ }^{1 \mathrm{le} / \mathrm{lle}}$ & $1.18(0.60-2.32)$ & $0.89(0.37-2.11)$ & $0.72(0.28-1.85)$ & $0.96(0.57-1.61)$ & $0.83(0.37-1.86)$ & $1.21(0.37-4)$ & $1.76(0.7-4.41)$ \\
\hline
\end{tabular}

Data are presented as odds ratios with 95\% confidence intervals. Models are adjusted for age, sex, parental education, parental asthma, parental atopy, gestational age, city, maternal smoking during pregnancy and environmental tobacco smoke at home. *: average days of incense burning at home per month.

burning are significantly associated with acute cough symptoms in primary school children.

In contrast, KoO et al. [19] have found that there is no association between incense burning and respiratory symptoms among primary school children and their nonsmoking mothers in Hong Kong. The age of the study population and the constituents of incense from different countries are all be possible contributors to discrepancies in findings.

The biological mechanisms by which the toxic effects of incense burning results in asthma are not well understood. It was reported that burning incense emitted $\mathrm{CO}, \mathrm{CO}_{2}, \mathrm{NO}_{2}, \mathrm{SO}_{2}$, DEP and PM. Exposures to $\mathrm{NO}_{2}$ and $\mathrm{SO}_{2}$ can lead to

TABLE 3 Association between incense burning at home and GSTT1 genotype on subcategories of asthma and wheeze

\begin{tabular}{|c|c|c|c|c|}
\hline & GSTT1 present & GSTT1 null & GSTT1 present & GSTT1 null \\
\hline \multicolumn{5}{|l|}{ Univariate model } \\
\hline \multicolumn{5}{|l|}{ Asthma } \\
\hline Lifetime asthma & 1.00 & $1.12(0.76-1.65)$ & $0.87(0.61-1.25)$ & $1.11(0.79-1.58)$ \\
\hline \multicolumn{5}{|l|}{ Wheeze } \\
\hline Lifetime wheeze & 1.00 & $1.08(0.78-1.48)$ & $0.87(0.65-1.17)$ & $1.12(0.84-1.49)$ \\
\hline Current wheeze & 1.00 & $0.96(0.57-1.62)$ & $0.79(0.49-1.27)$ & $1.03(0.65-1.63)$ \\
\hline Nocturnal wheeze $\mathrm{e}^{\#}$ & 1.00 & $0.62(0.28-1.36)$ & $0.84(0.45-1.58)$ & $1.19(0.65-2.16)$ \\
\hline Exercise wheeze & 1.00 & $1.27(0.75-2.14)$ & $1.67(1.06-2.62)$ & $1.68(1.07-2.66)$ \\
\hline \multicolumn{5}{|c|}{ Mutually adjusted model ${ }^{*}$} \\
\hline Medication use & 1.00 & $1.32(0.62-2.79)$ & $1.42(0.71-2.85)$ & $2.12(1.09-4.12)$ \\
\hline \multicolumn{5}{|l|}{ Wheeze } \\
\hline Lifetime wheeze & 1.00 & $1.08(0.77-1.50)$ & $1.02(0.75-1.39)$ & $1.27(0.94-1.72)$ \\
\hline Current wheeze $e^{\#}$ & 1.00 & $1.02(0.59-1.76)$ & $0.96(0.58-1.60)$ & $1.20(0.73-1.97)$ \\
\hline Nocturnal wheeze ${ }^{\#}$ & 1.00 & $0.65(0.29-1.45)$ & $0.87(0.44-1.70)$ & $1.30(0.69-2.46)$ \\
\hline Exercise wheeze & 1.00 & $1.33(0.77-2.28)$ & $1.89(1.17-3.05)$ & $1.91(1.18-3.09)$ \\
\hline
\end{tabular}

Data are presented as odds ratios with $95 \%$ confidence intervals. ${ }^{\#}$ : significant interaction; ${ }^{\prime}$ : models are adjusted for age, sex, parental education, parental asthma, parental atopy, gestational age, city, maternal smoking during pregnancy and environmental tobacco smoke at home. 


\begin{tabular}{|c|c|c|}
\hline \multirow[t]{2}{*}{ TABLE 4} & \multicolumn{2}{|c|}{$\begin{array}{l}\text { Association of frequency of incense burning at } \\
\text { home on subcategories of asthma and wheeze } \\
\text { by GSTT1 genotype }\end{array}$} \\
\hline & GSTT1 present & GSTT1 null \\
\hline Subjects $\mathbf{n}$ & 1937 & 1801 \\
\hline \multicolumn{3}{|c|}{ Frequency of incense burning ${ }^{\#}$} \\
\hline \multicolumn{3}{|c|}{ Asthma } \\
\hline \multicolumn{3}{|l|}{ Lifetime asthma } \\
\hline Never & 1.00 & 1.00 \\
\hline Less than daily & $0.74(0.45-1.21)$ & $0.74(0.45-1.22)$ \\
\hline Daily & $1.04(0.66-1.63)$ & $1.42(0.94-2.13)$ \\
\hline $\mathrm{p}$-value for trend" & 0.87 & 0.09 \\
\hline \multicolumn{3}{|l|}{ Current asthma } \\
\hline Never & 1.00 & 1.00 \\
\hline Less than daily & $1.22(0.60-2.50)$ & $1.05(0.54-2.05)$ \\
\hline Daily & $1.37(0.69-2.75)$ & $1.78(1.01-3.16)$ \\
\hline $\mathrm{p}$-value for trend" & 0.37 & 0.04 \\
\hline \multicolumn{3}{|l|}{ Medication use } \\
\hline Never & 1.00 & 1.00 \\
\hline Less than daily & $1.22(0.51-2.92)$ & $1.35(0.60-3.00)$ \\
\hline Daily & $1.37(0.59-3.18)$ & $2.26(1.11-4.57)$ \\
\hline $\mathrm{p}$-value for trend" & 0.47 & 0.02 \\
\hline \multicolumn{3}{|l|}{ Wheeze } \\
\hline \multicolumn{3}{|l|}{ Lifetime wheeze } \\
\hline Never & 1.00 & 1.00 \\
\hline Less than daily & $0.96(0.65-1.41)$ & $0.85(0.57-1.26)$ \\
\hline Daily & $1.02(0.70-1.49)$ & $1.40(1.00-1.98)$ \\
\hline$p$-value for trend" & 0.91 & 0.04 \\
\hline \multicolumn{3}{|l|}{ Current wheeze } \\
\hline Never & 1.00 & 1.00 \\
\hline Less than daily & $0.78(0.40-1.50)$ & $1.15(0.62-2.14)$ \\
\hline Daily & $1.01(0.55-1.87)$ & $1.17(0.65-2.11)$ \\
\hline$p$-value for trend" & 0.97 & 0.60 \\
\hline \multicolumn{3}{|l|}{ Nocturnal wheeze } \\
\hline Never & 1.00 & 1.00 \\
\hline Less than daily & $0.86(0.38-1.96)$ & $1.20(0.45-3.23)$ \\
\hline Daily & $0.85(0.37-1.95)$ & $3.21(1.41-7.30)$ \\
\hline $\mathrm{p}$-value for trend" & 0.69 & 0.003 \\
\hline \multicolumn{3}{|l|}{ Exercise wheeze } \\
\hline Never & 1.00 & 1.00 \\
\hline Less than daily & $2.03(1.16-3.54)$ & $1.28(0.71-2.31)$ \\
\hline Daily & $1.86(1.06-3.28)$ & $1.99(1.18-3.35)$ \\
\hline $\mathrm{p}$-value for trend" & 0.04 & 0.01 \\
\hline
\end{tabular}

Data are presented as odds ratios with $95 \%$ confidence intervals, unless otherwise stated. Models are adjusted for age, sex, parental education, parental asthma, parental atopy, gestational age, city, maternal smoking during pregnancy and environmental tobacco smoke at home. ${ }^{*}$ : average days of incense burning at home per month; ${ }^{\uparrow}$ : calculated by stratification with GSTT1 genotype.

respiratory illness, reduced pulmonary function and alterations in the lung's defence system [20]. Incense burning also produces volatile organic compounds, such as benzene, toluene, isoprene, xylenes, aldehydes and PAHs [6]. These compounds may lead to nose and throat irritation, asthma exacerbation and cancer.
Aside from irritated respiratory tracts, LIN et al. [21] also report that incense burning may cause elevated cord blood IgE. It is plausible that PM stimulates dendritic cells and T-cells to produce T-helper cell type 2 cytokines and activate proinflammatory genes in a process mediated by free radical and oxidative stress mechanisms [22]. Since exposure to lead can stimulate IgE production, it is speculated that lead emitted from incense burning first attaches to PM, subsequently transferring to fetal blood and modulating the fetal immune system with $\operatorname{IgE}$ production [21, 23]. Furthermore, incense smoke also causes morphological changes of pneumocytes and infiltrates of neutrophils in rat alveoli [24]. Activation of inflammatory cells may lead to amplification of various mediators, ending in inflammatory changes and airway remodelling [25].

Frequency of incense burning is associated with increased risk of asthma in an exposure-response manner in this study. After stratification by GSTT1 genotypes, the effect of incense burning remains only in the GSTT1 null genotype. GSTT1 also shows significant synergistic interaction with incense burning on current asthma, with OR 1.40 (95\% CI 0.76-2.57) for no incense exposure with GSTT1 null genotype, OR 1.36 (95\% CI 0.77-2.40) for incense exposure with GSTT1 present genotype and OR 1.92 (95\% CI 1.11-3.32) for incense exposure with GSTT1 null genotype. Previously, however, variants in the GSTM1 and GSTP1 loci reportedly contribute to the occurrence of childhood asthma, thereby increasing susceptibility to adverse effects of tobacco smoke $[10,14]$. The differences in genotype findings compared to other studies can be due to ethnic compositions and various distributions of genotype frequencies. There is a higher frequency of GSTT1 null genotypes in this study population than in Caucasians (48.1 versus $25.0 \%$ ) [26]. Such inconsistent results may underscore the inherent weakness of single-gene analyses for the study of geneenvironment interactions for a multigene disease like asthma. Since different polymorphisms of the GSTT1 gene have different effects on the detoxification ability of an individual, an incense smoke-induced airway injury is different in children carrying different GSTT1 polymorphisms. The statistically suggested interactions in this study add to the plausibility of a biological interaction between the GSTT1 enzyme and incense smoke in the detoxification process of reactive metabolic intermediates and reactive oxygen species [27, 28]. Furthermore, CORNELIS et al. [27] report that consumption of cruciferous vegetables is associated with lower risk of myocardial infarction only among those with a functional GSTT1 allele, which suggests that GSTT1 carriers can protect against oxidative stress or DNA damage [27, 29].

Consistent with previous studies, the GSTT1 null genotype is associated with increased risk of asthma [11, 30]. A possible explanation for this is that GST enzymes, largely expressed in human lung cells, act as detoxifying enzymes and serve as markers of putative oxidative stress [31]. Furthermore, altered antioxidant defences, lipid peroxidation and anti-inflammatory pathways are important in asthma pathogenesis [32]. It can be speculated that asthma caused by incense smoke partially contributes to DNA damage, which can occur from the lack of detoxification of reactive smoke metabolites by the GSTT1 enzyme [26, 33]. However, evidence for an association between GSTs and asthma is inconsistent. The findings of MinELLI et al. [34] 
do not support a substantial role of GST genes alone in the development of asthma. Future large-scale studies about interactions of GST genes with environmental oxidative exposures and other genes involved in antioxidant pathways among genetically susceptible individuals are necessary.

This study has some potential limitations that may influence the interpretation of the results. First, the degree of ventilation of the room where incense was burned is not known. The amount of incense smoke inhaled will be biased, because incense exposure has been calculated based on the questionnaire. There is also no good quantitative biomarker for incense burning presently. Exposure assessments from the questionnaire are regarded as appropriate surrogate measurements of incense smoke exposure, which may be subject to misclassification bias. Furthermore, the study subjects are likely to be exposed to other sources of incense aside from their own houses. If incense smoke exposure from temples or other places are included in the analyses, the current amount of incense smoke used will most likely double or triple. Misclassification bias will then shift the results toward the null. Another possible limitation is recall bias in respiratory outcomes. The recall of asthma status was assessed in a subset of the study population and found that the concordance of parental reports of asthma and medical records' documentation of asthma was good.

One of the strengths of this study is its inclusion of a large and sociodemographically diverse population of children in Taiwan. Unbiased observations of the association between genetic polymorphisms and outcomes are expected. In addition, the association between incense burning has been investigated with many respiratory outcomes. To our knowledge, the interaction of genetic polymorphisms and incense burning on respiratory manifestations has not been previously reported. The results reported here suggest that genotyping for GSTT1 polymorphism, a simple and inexpensive assay, may be a suitable biomarker for identifying genetically susceptible children.

In conclusion, household incense exposure has adverse effects on children carrying the GSTT1 null genotypes. These findings not only help us to understand the aetiology of asthma but also guide potential control measures in the future. The diverse ability to detoxify incense smoke depends on variations of GSTT1 polymorphisms. As incense smoke is a complex mixture of chemicals, and since other metabolic genes may be involved, additional long-term research is needed to explore the relative role of other genes in determining genetic susceptibility to adverse respiratory outcomes.

\section{SUPPORT STATEMENT}

This study was supported by the grant \#98-2811-B-002-137 and \#962314-B-006-053 from Taiwan National Science Council and Department of Health in Taiwan. The funding source had no role in the design or analysis of the study.

\section{STATEMENT OF INTEREST}

None declared.

\section{ACKNOWLEDGEMENTS}

The authors thank all of the field workers who supported the data collection, the school administrators and teachers, and especially the parents and children who participated in this study.

\section{REFERENCES}

1 Yan DC, Ou LS, Tsai TL, et al. Prevalence and severity of symptoms of asthma, rhinitis, and eczema in 13- to 14-year-old children in Taipei, Taiwan. Ann Allergy Asthma Immunol 2005; 95 : 579-585.

2 Anderson HR, Ruggles R, Strachan DP, et al. Trends in prevalence of symptoms of asthma, hay fever, and eczema in 12-14 year olds in the British Isles, 1995-2002: questionnaire survey. BMJ 2004; 328: 1052-1053.

3 Douwes J, Pearce N. Asthma and the westernization "package". Int J Epidemiol 2002; 31: 1098-1102.

4 Wen CP, Levy DT, Cheng TY, et al. Smoking behaviour in Taiwan, 2001. Tob Control 2005; 14: Suppl. 1, i51-i55.

5 Environmental Protection Bureau. Total Inventory Control of Air Pollutants and the Guidance Program for Reduction. Kaohsiung, Kaohsiung City Government, 2003.

6 Lin TC, Krishnaswamy G, Chi DS. Incense smoke: clinical, structural and molecular effects on airway disease. Clin Mol Allergy 2008; 6: 3.

7 Abdul WA, Mostafa OA. Arabian incense exposure among Qatari asthmatic children. A possible risk factor. Saudi Med J 2007; 28: 476-478.

8 Chiang KC, Liao CM. Heavy incense burning in temples promotes exposure risk from airborne PMs and carcinogenic PAHs. Sci Total Environ 2006; 372: 64-75.

9 Sampsonas F, Archontidou MA, Salla E, et al. Genetic alterations of glutathione $S$-transferases in asthma: do they modulate lung growth and response to environmental stimuli? Allergy Asthma Proc 2007; 28: 282-286.

10 Hanene C, Jihene L, Jamel A, et al. Association of GST genes polymorphisms with asthma in Tunisian children. Mediators Inflamm 2007; 2007: 19564.

11 Kabesch M, Hoefler C, Carr D, et al. Glutathione S transferase deficiency and passive smoking increase childhood asthma. Thorax 2004; 59: 569-573.

12 Saadat M, Ansari-Lari M. Genetic polymorphism of glutathione $S$ transferase T1, M1 and asthma, a meta-analysis of the literature. Pak J Biol Sci 2007; 10: 4183-4189.

13 Piacentini S, Polimanti R, Moscatelli B, et al. Glutathione Stransferase gene polymorphisms and air pollution as interactive risk factors for asthma in a multicentre Italian field study: a preliminary study. Ann Hum Biol 2010; 37: 427-439.

14 Romieu I, Ramirez-Aguilar M, Sienra-Monge JJ, et al. GSTM1 and GSTP1 and respiratory health in asthmatic children exposed to ozone. Eur Respir J 2006; 28: 953-959.

15 Tsai $\mathrm{CH}$, Huang JH, Hwang BF, et al. Household environmental tobacco smoke and risks of asthma, wheeze and bronchitic symptoms among children in Taiwan. Respir Res 2010; 11: 11.

16 Lee YL, Hsiue TR, Lee YC, et al. The association between glutathione S-transferase P1, M1 polymorphisms and asthma in Taiwanese schoolchildren. Chest 2005; 128: 1156-1162.

17 Al-Rawas OA, Al-Maniri AA, Al-Riyami BM. Home exposure to Arabian incense (bakhour) and asthma symptoms in children: a community survey in two regions in Oman. BMC Pulm Med 2009; 9: 23.

18 Yang CY, Chiu JF, Cheng MF, et al. Effects of indoor environmental factors on respiratory health of children in a subtropical climate. Environ Res 1997; 75: 49-55.

19 Koo LC, Ho JC, Tominaga S, et al. Is Chinese incense smoke hazardous to respiratory health? Indoor Built Environ 1995; 4: 334-343.

20 Bernard SM, Samet JM, Grambsch A, et al. The potential impacts of climate variability and change on air pollution-related health effects in the United States. Environ Health Perspect 2001; 109: Suppl. 2, 199-209.

21 Lin YC, Wen HJ, Lee YL, et al. Are maternal psychosocial factors associated with cord immunoglobulin $\mathrm{E}$ in addition to family 
atopic history and mother immunoglobulin E? Clin Exp Allergy 2004; 34: 548-554.

22 Donaldson K, Stone V, Borm PJ, et al. Oxidative stress and calcium signaling in the adverse effects of environmental particles (PM10). Free Radic Biol Med 2003; 34: 1369-1382.

23 Lutz PM, Wilson TJ, Ireland J, et al. Elevated immunoglobulin E (IgE) levels in children with exposure to environmental lead. Toxicology 1999; 134: 63-78.

24 Wegmann M. Th2 cells as targets for therapeutic intervention in allergic bronchial asthma. Expert Rev Mol Diagn 2009; 9: 85-100.

25 Alarifi SA, Mubarak MM, Alokail MS. Ultrastructural changes of pneumocytes of rat exposed to Arabian incense (bakhour). Saudi Med J 2004; 25: 1689-1693.

26 Nukui T, Day RD, Sims CS, et al. Maternal/newborn GSTT1 null genotype contributes to risk of preterm, low birthweight infants. Pharmacogenetics 2004; 14: 569-576.

27 Cornelis MC, El-Sohemy A, Campos H. GSTT1 genotype modifies the association between cruciferous vegetable intake and the risk of myocardial infarction. Am J Clin Nutr 2007; 86: 752-758.

28 See SW, Wang YH, Balasubramanian R. Contrasting reactive oxygen species and transition metal concentrations in combustion aerosols. Environ Res 2007; 103: 317-324.
29 Dellinger B, Pryor WA, Cueto R, et al. Role of free radicals in the toxicity of airborne fine particulate matter. Chem Res Toxicol 2001; 14: 1371-1377.

30 Vavilin VA, Makarova SI, Liakhovich VV, et al. Polymorphic genes of xenobiotic-metabolizing enzymes associated with predisposition to bronchial asthma in hereditarily burdened and nonburdened children. Rus J Genet 2002; 38: 439-445.

31 Ercan H, Birben E, Dizdar EA, et al. Oxidative stress and genetic and epidemiologic determinants of oxidant injury in childhood asthma. J Allergy Clin Immunol 2006; 118: 1097-1104.

32 Altinisik J, Balta ZB, Aydin G, et al. Investigation of glutathione Stransferase M1 and T1 deletions in lung cancer. Mol Biol Rep 2010; 37: 263-267.

33 Popp W, Vahrenholz C, Schell C, et al. DNA single strand breakage, DNA adducts, and sister chromatid exchange in lymphocytes and phenanthrene and pyrene metabolites in urine of coke oven workers. Occup Environ Med 1997; 54: 176-183.

34 Minelli C, Granell R, Newson R, et al. Glutathione-S-transferase genes and asthma phenotypes: a Human Genome Epidemiology (HuGE) systematic review and meta-analysis including unpublished data. Int J Epidemiol 2010; 39: 539-562. 